\title{
Phase Equilibria Calculations of Polyethylene Solutions from SAFT-Type Equations of State
}

\author{
Nuno Pedrosa, ${ }^{\dagger}$ Lourdes F. Vega, ${ }^{\dagger}$ João A. P. Coutinho, ${ }^{\dagger}$ and Isabel M. Marrucho $*, \dagger$ \\ CICECO, Departamento de Química, Universidade de Aveiro, 3810 Aveiro, Portugal, and Institut de \\ Ciència de Materials de Barcelona (ICMAB-CSIC), Consejo Superior de Investigaciones Científicas, \\ Campus de la U.A.B., 08193 Barcelona, Spain
}

Received March 15, 2006; Revised Manuscript Received April 18, 2006

\begin{abstract}
We present here phase equilibria calculations of polyethylene solutions in different solvents as obtained with two versions of the SAFT equation of state, soft-SAFT and PC-SAFT. The objective of this work is twofold: to check the accuracy of the soft-SAFT equation in providing reliable polymer solutions behavior and to propose a methodology from which systematic studies on polymer solutions can be made by the use of transferable molecular parameters. Some issues regarding the fitting of molecular parameters from polymer data as well as the numerical problems associated with polymer phase equilibria calculations are also mentioned. The methodology is applied to model the phase equilibria of polyethylene solutions with several solvents, differing in size and polarity, including $n$-pentane, $n$-hexane, butyl acetate, and pentanol, and results are compared to available experimental data. The phase behavior explored in this work is wide, from vapor-liquid equilibria to liquid-liquid equilibria, displaying upper critical solution temperatures and lower critical solution temperatures. We have also calculated the solubility of ethylene in polyethylene with the same models. Results obtained from the soft-SAFT equation are slightly more accurate in some of the cases than the PC-SAFT equation. Both equations, soft-SAFT and PC-SAFT, follow most of the experimental trends, providing accurate predictions from pure component parameters in some of the cases, while a binary interaction parameter was needed for the butyl acetate, 1-pentanol, and ethylene binary mixtures.
\end{abstract}

\section{Introduction}

Although polymers are found in a wide spread range of applications, the modeling of phase equilibria of polymers systems still remains a challenging task. The increasing complexity of polymers and polymer systems resulting from new polymerization techniques and the new approaches to their use aggravates this situation. From a past situation where polymers were used in an almost pure state, i.e., few additives were used to improve their chemical and mechanical properties, to the present situation where the polymeric material properties can be tailored to specification by formulation, polymer phase equilibria have increased in complexity but also in importance. The absence of adequate models for the polymer system properties and phase behavior makes this design procedure a time-consuming and costly task that is performed on a trialand-error basis with more art and skillful judgment than solid science.

The polymer-solvent phase equilibria is, in fact, a very important aspect in the manufacturing, processing, and formulation of polymers. In the polymerization reaction there are several compounds to consider, depending on the type of reaction, such as polymer, unreacted monomer, and often solvent. The other compounds that might be present (initiator, surfactant, etc.) can be neglected in terms of phase equilibria as their amount is usually too small to influence it significantly. The processing of the polymer also involves phase equilibria calculations, as the unreacted monomer and the solvent have to be separated from the polymer, usually via flash drums.

Polymer-solvent solutions usually exhibit fluid phase equilibria of type IV and V according to the classification of Scott

\footnotetext{
Universidade de Aveiro.

Consejo Superior de Investigaciones Científicas.

* Corresponding author: e-mail imarrucho@dq.ua.pt.
}

and van Konynenburg. ${ }^{1}$ The characteristic of these mixtures is the existence of a lower critical end point (LCEP) and an upper critical end point (UCEP). The occurrence of these critical points is due to the large difference of sizes between the two molecules, leading to a large difference in their volatility. The combination of these factors leads to phase split in which three phases may coexist: two liquid phases and one gas phase.

The usual approach to the modeling of these complex systems falls in two main groups: on one hand, there are the free energy models (Flory-Huggins, NRTL, UNIFAC, and UNIFAC-FV) and, on the other hand, the equation of state based models (Sanchez-Lacombe, polymer-SRK, SAFT). The most successful one in terms of widespread use is the Flory-Huggins model, developed from the lattice fluid theory. ${ }^{2}$ Its success comes from its mathematical simplicity when compared to equations of state, while the results produced are quite acceptable for several common polymer systems. The Sanchez-Lacombe equation of state (EoS), ${ }^{3,4}$ also developed from the lattice fluid theory, has also been quite successful in modeling vapor-liquid equilibria and liquid-liquid equilibria of polymer systems. ${ }^{5,6}$

One approach that is rising in popularity, due to its accuracy, is the estimation of thermodynamic properties of polymer solutions by the statistical association fluid theory (SAFT). The SAFT equation is based on Wertheim's (TPT1) theory, ${ }^{7-10}$ and it was later converted into an useful model by Chapman et al. ${ }^{11}$ The underlying concept behind SAFT is its description of the molecules of interest. In the SAFT approach, the individual molecules are "constructed" by the addition of different terms: the reference term, the chain term, and the association term. The reference term is usually a spherical segment, which can be a Lennard-Jones, a hard sphere, and even a square well fluid. These segments are then linked together to make the molecular chains present in the fluid. This concept is the reason why this 
EoS seems to be appropriate to describe the phase equilibria of long chain molecules, such as polymers. If the molecules are associating (i.e., they are able to form hydrogen bonds), an additional term is added to take into account this contribution. Several versions of SAFT have been developed, mostly differing in the reference term used. ${ }^{11-15}$

The use of the SAFT EoS in modeling the polymer phase equilibria comes from its debut. Huang and Radosz ${ }^{12}$ first presented the modeling of pure polymers with this approach; i.e., only the pure polymer molecular parameters were presented without any modeling of mixtures. Huang and Radosz obtained the molecular parameters of pure polymers by fitting merely to the polymers' densities, as the polymers have no measurable vapor pressure. The first successful modeling of polymer mixtures with the SAFT EoS reported in the literature was done by Chen et al., ${ }^{16}$ based on the initial suggestion of the previously mentioned work that polymer mixtures could be modeled with the original SAFT EoS. The original SAFT EoS showed very good results in the modeling of mixtures of poly(ethylenepropylene) with some solvents. Following this work, Wu and Chen, ${ }^{17}$ Ghonasgi and Chapman, ${ }^{18}$ and Koak and Heidemann ${ }^{19}$ successfully applied the SAFT EoS to the modeling of polymer solutions, in particular to the liquid-liquid equilibria presented by these types of systems.

Recently, Gross and Sadowski ${ }^{15}$ have developed a variation of the SAFT model (PC-SAFT) in which the reference term is a hard chain fluid instead of a hard sphere fluid. This feature makes this equation very attractive to model polymer phase equilibria since the particular connection between the different segments is already taken into account in the reference term. In fact, at present time PC-SAFT is the most used version of the SAFT EoS for polymers. ${ }^{20,21}$ In this context, von Solms et al. ${ }^{22}$ recently proposed a simplification in the mixing rules to lower the computing time of phase equilibria calculations with this approach. This model has been applied to a number of system types involving polymer phase equilibria. ${ }^{23-26}$

The modeling of polymer phase equilibria with SAFT-type equations and related approaches poses two problems: the calculation of the polymers pure component molecular parameters, due to the lack of vapor pressure in polymer melts, and the numerical problems due to the large asymmetry of polymersolvent/gas systems. The fitting of SAFT parameters for polymers is a very debated problem: ${ }^{20,24}$ the problem is that the parameters obtained by fitting only to the density data of pure polymers provide poor descriptions of the mixtures behavior, hence lacking transferability. One of the proposed solutions was to fit the polymer parameters to a polymer binary mixture. ${ }^{20}$ This is a rather controversial procedure, as the fitted parameters may be suitable just for that system and may not be transferable to other related binaries with the same polymer, but with others solvents that have different characteristics, like for instance associating compounds. Another question that arises from this procedure is how much of the binary interaction parameter is incorporated into the polymer parameters, and vice versa, how much of the polymer parameters is incorporated into the binary interaction parameter?

The scope of this work is to develop a model based on the soft-SAFT EoS, proposed by Blas and Vega ${ }^{14}$ for the description of vapor-liquid and liquid-liquid equilibria of polymersolvent systems and, in addition, to provide a methodology in which systematic studies on polymer solutions can be made by the use of transferable molecular parameters with molecularbased EoS. The performance of the soft-SAFT equation is compared with the PC-SAFT EoS, since PC-SAFT has been widely used for polymeric systems. Both equations proceed from a thermodynamic perturbation theory in which different perturbation terms are added to a reference term. The main difference between this two EoS's is the reference term. Both equations have proved to be accurate for phase equilibria calculations of several pure fluids and binary mixtures. While most of the applications of PC-SAFT deal with polymeric systems, the use of the soft-SAFT equation to long chain molecules has been very limited until the present study. In fact, regarding the calculation of thermodynamic properties of long chain molecules, soft-SAFT has been applied, so far, to the calculation of critical points of homopolymer systems ${ }^{27}$ and to study the solubility of hydrogen in heavy $n$-alkanes, ${ }^{28}$ obtaining good agreement with available simulation and experimental data. In both cases, the molecular parameters were calculated from correlations obtained as a function of the molecular weight of the compounds obtained by fitting the first eight members of the $n$-alkane series. ${ }^{29}$ Our objective here is to check the performance of this model for polymer solutions with different solvents. The comparison with PC-SAFT predictions, with no fitting of binary parameters, will show the robustness of the soft-SAFT EoS to model polymer solutions and the accuracy of both equations for this purpose.

The rest of the paper is organized as follows. Section 2 is devoted to the modeling approach described here. Results and discussions are presented in section 3, while some concluding remarks are provided in the last section.

\section{Modeling}

The SAFT approach involves a sum of molecular contributions in order to describe a fluid. In most SAFT equations these molecular contributions are expressed in terms of residual Helmholtz energy as follows:

$$
A^{\text {res }}=A-A^{\text {ideal }}=A^{\text {ref }}+A^{\text {chain }}+A^{\text {assoc }}
$$

where $A^{\text {ideal }}, A^{\text {ref }}, A^{\text {chain }}$, and $A^{\text {assoc }}$ stand for the ideal, reference, chain, and association contribution to the total Helmholtz energy.

As was already mentioned, different reference terms have been proposed in the development of SAFT EoSs, such as the Lennard-Jones, ${ }^{14}$ the hard sphere, ${ }^{12}$ or the square-well ${ }^{13}$ potentials. In the case of the hard sphere reference term an extra contribution should be included in eq 1 to take into account the dispersive interactions. In eq 1 , the chain term accounts for the energy required to make a chain molecule out of the segments of the reference fluid to produce the desired molecule. The association term is used when physical or more usually chemical association between molecules is present in the system. Both terms come from Wertheim's theory for associating fluids. For details on the formulation and implementation, the reader is referred to two reviews on the subject. ${ }^{30,31}$

As stated in the Introduction, two versions of the SAFT EoS are used: soft-SAFT ${ }^{14,29}$ and PC-SAFT. ${ }^{20}$ Soft-SAFT uses a Lennard-Jones (LJ) spherical fluid as the reference term, including attractive and repulsive interactions in this term, while the reference term in the PC-SAFT equation is a hard-sphere chain. The chain contribution is included in soft-SAFT as a perturbation to the reference term, from Wertheim's theory. The radial distribution function of the $\mathrm{LJ}$ fluid is used to obtain the final expression for the chain term. In the case of PC-SAFT, the dispersive interactions are included as a perturbative term, while the radial distribution function in the chain term is already that of a chain, instead of segments. The complete expressions 
for each version are provided in the original references, and the reader is referred to them for details. ${ }^{14,20}$

Having the model thus defined, molecular parameters are needed to define the uniqueness of each fluid in terms of the SAFT EoS description. The soft-SAFT model, which is described in detail in the literature, ${ }^{14,29}$ has three parameters for nonassociating fluids: the size of LJ singular segments, $\sigma$, the energy of the segments, i.e., the well-depth of the LJ potential, $\epsilon$, and the number of segments, $m$. For associating fluids, two more parameters are required: the association energy, $\epsilon_{\mathrm{HB}}$, and the volume of association, $k_{\mathrm{HB}}$. In most applications of soft-SAFT to experimental systems these parameters were estimated by fitting the pure component vapor pressure and saturated liquid densities simultaneously. The application of this equation to mixtures may require the use of one or two extra binary interaction parameters, which account for asymmetries in the energies of the components, $\xi_{i j}$, or their sizes, $\eta_{i j}$, according to the generalized Lorentz-Berthelot combining rules. ${ }^{14}$

$$
\begin{gathered}
\epsilon_{i j}=\xi_{i j}\left(\epsilon_{i i} \epsilon_{j j}\right)^{1 / 2}=\left(1-k_{i j}\right)\left(\epsilon_{i i} \epsilon_{j j}\right)^{1 / 2} \\
\sigma_{i j}=\eta_{i j}\left(\frac{\sigma_{i i}+\sigma_{j j}}{2}\right)
\end{gathered}
$$

Concerning polymer phase equilibria calculations, the component's composition is usually presented as mass fractions, since the polymer has a much higher molecular weight than the solvent. The SAFT equation of state is defined in terms of molar fractions, which might present a problem when computing the solvent molar fraction as they might become very small, leading to numerical problems. A similar problem may occur when modeling polymer vapor-liquid equilibria: in the gas phase there is virtually no polymer, but thermodynamically this value cannot be zero, even tough it might be extremely low. This produces some difficulties in calculating phase equilibria via flash calculations. The asymmetry of the system leads to very low fugacities which may go past the underflow of the computer system. A solution to this problem was proposed by Ghosh and co-workers, ${ }^{32}$ who assured the thermodynamics stability by using the tangent plane distance method. In the present work, the phase equilibria coexistence in equilibrium is assured by the equality of chemical potentials of each component in each phase as well as the total pressure of each phase. Hence, the calculations presented here were performed using bubble pressure calculations and dew point calculations.

\section{Results and Discussion}

Polyethylene-solvent/gas-phase behavior was chosen to evaluate the performance of the soft SAFT EoS in the description of both VLE/GLE and LLE for polymeric systems. One of the reasons for choosing this polymer was the existence of a correlation of parameters for the $n$-alkane series; ${ }^{29}$ as polyethylene can be considered as a very large alkane, it would be possible to extrapolate these correlations for higher molecular weight molecules. Also, a series of solvents were chosen in order to obtain a rich phase behavior, from vapor-liquid to liquidliquid equilibria, with the aim of extensively evaluating the performance of the soft-SAFT model for polymer solutions.

3.1. Pure Polyethylene Parameters. To adequately describe the phase behavior of a mixture with the soft-SAFT equation of state, the molecular parameters for the pure compounds need to be estimated first. Because of the absence of vapor pressure of polymer melts, two different approaches are used here.

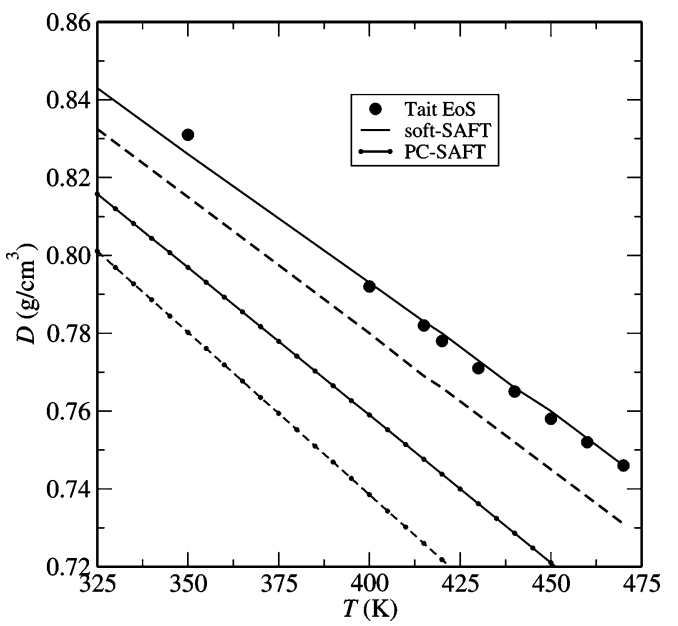

Figure 1. Polymer melt density of a polyethylene with a $M_{\mathrm{n}}=16000$ at a pressure of 0.1 MPa. Dots are some values calculated with the Tait EoS. ${ }^{33}$ The full lines are calculated with both soft-SAFT and PCSAFT EoS models using correlation of parameters for the $n$-alkanes series (see Table 1); the dashed lines are the calculated densities using correlation of parameters developed in this work for the soft-SAFT and the parameters from literature for PC-SAFT. ${ }^{20}$ Lines with small full circles correspond to PC-SAFT calculations.

First, polyethylene molecular parameters were obtained by extrapolation of the correlation of parameters with molecular weight from the $n$-alkane series: ${ }^{29}$

$$
\begin{gathered}
m=0.0255 M_{\mathrm{n}}+0.628 \\
m \sigma^{3}=1.73 M_{\mathrm{n}}+22.8 \\
m \epsilon / k_{\mathrm{B}}=7.89 M_{\mathrm{n}}+38.0
\end{gathered}
$$

where $m$ is the length of the chain of segments, $\sigma$ is the size of each segment (LJ diameter), $\epsilon$ is the energy of the segments, and $M_{\mathrm{n}}$ is the number molecular weight (in $\mathrm{g} / \mathrm{mol}$ ). Units of $\sigma$ and $\epsilon / k_{\mathrm{B}}$ are in $\AA$ and $\mathrm{K}$, respectively. We have derived a similar correlation for the PC-SAFT equation using the molecular parameters given by the authors, ${ }^{20}$ in a similar way as done by Pàmies and Vega: ${ }^{29}$

$$
\begin{gathered}
m=0.0257 M_{\mathrm{n}}+0.844 \\
m \sigma^{3}=1.73 M_{\mathrm{n}}+19.36 \\
m \epsilon / k_{\mathrm{B}}=7.01 M_{\mathrm{n}}+109.44
\end{gathered}
$$

The notation is the same as in eq 2 . These correlations make possible the calculation of molecular parameters for polyethylene with different molecular weights.

Figure 1 depicts the density behavior with respect to temperature of a linear polyethylene with $M_{\mathrm{n}}=16000$ calculated with the correlations of eqs 2 and 3 for the softSAFT and PC-SAFT EoS's, respectively. The results obtained are compared to those obtained by the Tait equation using parameters found in the literature. ${ }^{33}$ The prediction of the density dependence with temperature calculated with the soft-SAFT EoS using the extrapolation of the molecular parameters correlation for the alkane series ${ }^{29}$ agrees very well with the one predicted by the Tait equation. The average absolute deviation (AAD) is of $0.14 \%$. Although results for the pure polymer density are excellent, the results obtained for mixtures were not as accurate, as will be shown later. This has been previously observed when applying SAFT-type equations to polymer solutions. The most 
common approach to solve this is problem is to fit the polymer parameters to a polymer mixture and then to use the new parameters to model that polymer's mixture. We took a different approach in this work: instead of fitting to a mixture, a new correlation for the energy parameter was derived, while the expressions for the chain length $(m)$ and segment size $(\sigma)$ remained the same as in eq 2 . The resulting expression for the energy has the following form:

$$
\epsilon / k_{\mathrm{B}}=\frac{294.9 M_{\mathrm{n}}-779.38}{M_{\mathrm{n}}+10.6643}
$$

As in the original work of Pàmies and Vega, ${ }^{29}$ this equation was obtained by fitting the values of energy and molecular weight of the alkane series from methane to $n$-octane. Emphasis was put on accurately describing the heavier members of the series, instead of obtaining an excellent overall agreement for all members of the series. With this expression the limiting value of the energy term is lowered to a value of $294.9 \mathrm{~K}$ in comparison with the one given by eq 2c, which is $309.4 \mathrm{~K}$. Although the difference between these two limiting values is not large, the fact that this parameter value is repeated $m$ times for each polymer molecule produces great differences in the final results, as will be shown later. A main advantage of this approach vs fitting to a mixture is that they are pure component parameters which can be used to describe several mixtures, without using any mixture information in the fitting procedure, in a predictive manner. The results for the pure polymer density obtained for linear polyethylene with $M_{\mathrm{n}}=16000$ with this new correlation are also displayed in Figure 1. Interestingly, the prediction of the pure polymer density using eq 4 yields an AAD of $1.7 \%$, which is larger than the one obtained when eq $2 \mathrm{c}$ is used. However, the accuracy of the equation for the description of polyethylene mixtures is better, as will be shown in the next section.

Predictions of the polymer densities using PC-SAFT are also presented in Figure 1, for comparative purposes. In a similar way, two sets of molecular parameters were used for these calculations. The full line represents results obtained with parameters extrapolated from the correlation for the $n$-alkane series (eq 3), while the dashed line represents results with the parameters of linear polyethylene which were fitted to the mixture of polyethylene with ethane by the authors. ${ }^{15}$ Note that this is not the approach we have taken with the soft-SAFT equation here. Results obtained with the PC-SAFT EoS and both sets of parameters are not as accurate as those obtained with the soft-SAFT EoS, showing AADs of $4.0 \%$ and $8.0 \%$, respectively.

The molecular parameters of the pure compounds used in this work are listed in Tables 1 (polymers) and 2 (solvents), along with their respective sources.

3.2. Phase Equilibria Modeling. The performance of the chosen equation and was investigated for a wide range of phase diagrams. We have modeled different types of polyethylene mixtures, ranging from liquid-liquid mixtures to vapor-liquid equilibrium mixtures and gas solubility. The solvents studied include nonassociating compounds such as alkanes and alkenes, polar compounds such as esters, and associating compounds such as alcohols.

3.2.1. Polyethylene $/ \boldsymbol{n}$-Pentane. Predictions for the liquidliquid phase equilibria diagram for the system of polyethylene, $M_{\mathrm{n}}=16000 \mathrm{~g} / \mathrm{mol}$ and $M_{\mathrm{w}} / M_{\mathrm{n}}=1.16$, with $n$-pentane at a pressure of 5 and $10 \mathrm{MPa}^{34}$ calculated using both sets of softSAFT and PC-SAFT polymer parameters are presented in Figure

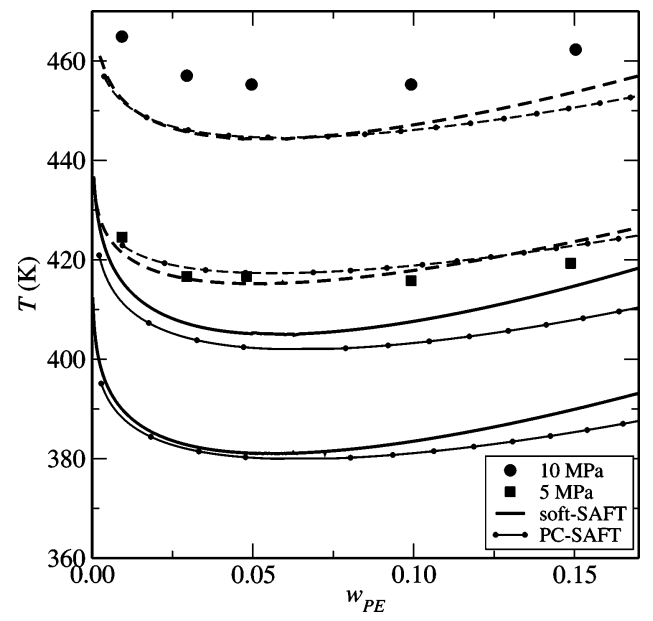

Figure 2. Liquid-liquid equilibria of polyethylene (16 000) and $n$-pentane using soft-SAFT and PC-SAFT. Line description as in Figure 1. Experimental data from Kiran and Zhuang. ${ }^{34}$

2. Calculations were performed with the molecular parameters of the pure components, in a predictive manner; i.e., no binary interaction parameters were used. The advantage of using the new correlation proposed in eq 4 is clearly depicted in Figure 2 , where a better description of the phase behavior of the mixture at two different pressures is observed. The results obtained with soft-SAFT are equivalent to those obtained using the PC-SAFT with molecular parameters for polyethylene proposed by Gross and Sadowski, which were calculated by fitting them to a mixture of polyethylene and ethene. ${ }^{20}$ It is interesting to remark that despite the differences between the two SAFT EoS in predicting the density of the pure polymer (see Figure 1), both equations behave in a very similar way when describing LLE for mixtures.

Modeling the vapor-liquid equilibria of polymer solutions is a challenge also from the numerical point of view. In this type of phase equilibria, no polymer molecules are present in the gas phase, as it does not have a measurable vapor pressure; only the solvent is present. Thus, the actual molar fraction of the polymer in the gas phase is virtually zero, but thermodynamically it should have a finite value close to zero. Predictions of vapor-liquid equilibrium for a pentane-polyethylene $\left(M_{\mathrm{n}}\right.$ $=76000 \mathrm{~g} / \mathrm{mol}$ ) mixture ${ }^{35}$ at two different temperatures are shown in Figure 3. The polymer molecular parameters used in the soft-SAFT EoS were obtained from eq 4. For comparative purposes, we also show the description of the PC-SAFT model. As in Figure 2, no binary interaction parameters were used; results for the mixture were obtained from pure component parameters. The results obtained are in excellent agreement with the experimental data, indicating that both SAFT models provide a good description of both polymer vapor-liquid and liquidliquid equilibrium using these parameters.

3.2.2. Polyethylene $/ \boldsymbol{n}$-Hexane. Chen et al. ${ }^{36}$ measured the liquid-liquid equilibria of polyethylene and $n$-hexane. The polymer was bimodal with number molecular weights of 15000 and $108000 \mathrm{~g} / \mathrm{mol}$. We have modeled this bimodal polymer as a mixture of two polymers, which in this case is a polymer equimolar mixture. The two separated polymer binary systems, polyethylene $\left(M_{\mathrm{n}}=15000 \mathrm{~g} / \mathrm{mol}\right) / n$-hexane and polyethylene $\left(M_{\mathrm{n}}=108000 \mathrm{~g} / \mathrm{mol}\right) / n$-hexane, were also modeled, and the results are shown in Figures 4 and 5, respectively. Again, the polymer molecular parameters were obtained from eq 4 , and no binary interaction parameters were used. Note that for the heavier polymer the critical point of the mixture is well predicted but the subcritical region is underestimated, while for the lower 


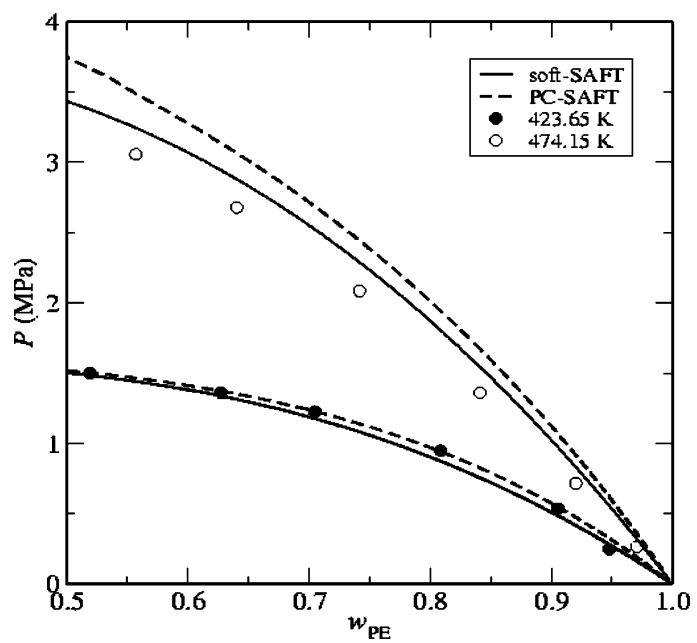

Figure 3. Modeling of the isothermal vapor-liquid equilibria of polyethylene $\left(M_{\mathrm{n}}=76000\right)$ and $n$-pentane with the soft-SAFT EoS (full lines) and with the PC-SAFT (dashed lines) EoS. Experimental data from Surana et al. ${ }^{35}$

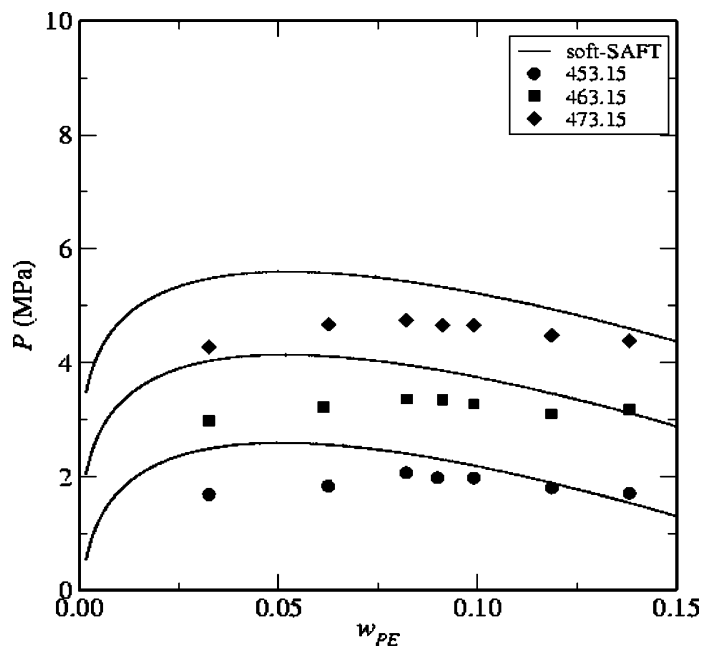

Figure 4. Liquid-liquid equilibria of a mixture of polyethylene $\left(M_{\mathrm{n}}\right.$ $=15000)$ and $n$-hexane at isothermal conditions: line, soft-SAFT predictions; symbols, experimental data taken from the literature. ${ }^{36}$

molecular weight polymer the critical point is overestimated. The bimodal polymer modeling results are shown in Figure 6 at $453.15 \mathrm{~K}$. The phase equilibrium description for the bimodal mixture is well achieved by the soft-SAFT model, especially considering that this is a very challenging system and the curves correspond to pure predictions.

3.2.3. Polyethylene/Butyl Acetate. Besides mixtures with just an UCST or a LCST, it is also of interest to describe systems with two (LCST and UCST) critical points. Figure 7 shows the soft-SAFT description of the mixture of polyethylene $\left(M_{\mathrm{n}}=\right.$ 64000 and $M_{\mathrm{n}}=13600 \mathrm{~g} / \mathrm{mol}$ ) and butyl acetate where the experimental data were taken from Kuwahara et al. ${ }^{37}$ This mixture shows an UCST and a LCST behavior, being a challenging mixture to any modeling approach. Calculations made with pure component parameters (as done in the previous cases) lead to an extremely low underprediction, giving a UCST of around $150 \mathrm{~K}$ when the experimental value is around $420 \mathrm{~K}$ for the lower molecular weight polymer. The use of one binary interaction parameter was needed to obtain a reasonable description of the low-temperature region, although no influence of this parameter was observed for the high-temperature region. This fact leads to the conclusion that the lower temperature phase coexistence diagram is driven by the energy of interaction

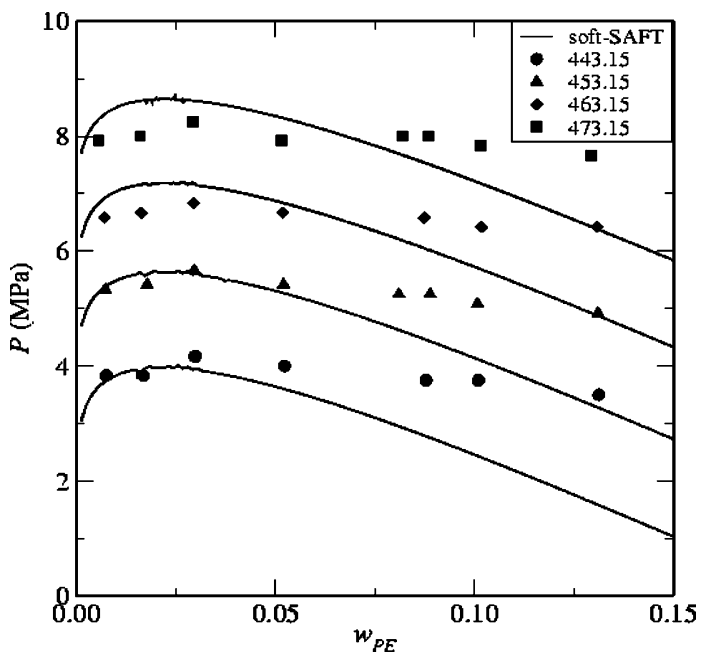

Figure 5. Liquid-liquid equilibria of a mixture of polyethylene $\left(M_{\mathrm{n}}\right.$ $=108000)$ and $n$-hexane at isothermal conditions: line, soft-SAFT predictions; symbols, experimental data taken from the literature. ${ }^{36}$

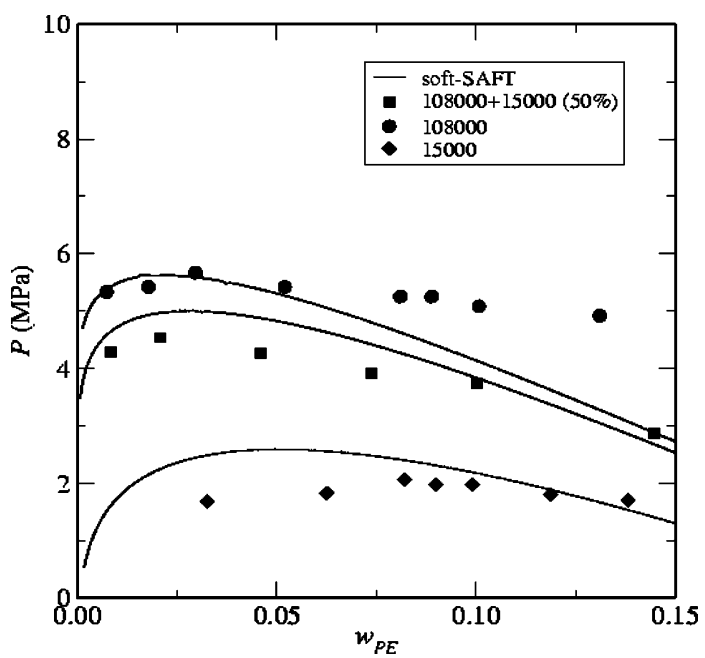

Figure 6. Liquid-liquid equilibria of a mixture of a bimodal polyethylene $\left(M_{\mathrm{n} 1}=15000\right.$ and $\left.M_{\mathrm{n} 2}=108000\right)$ and $n$-hexane at isothermal conditions. Comparison with the pure polyethylenes of molecular weights 15000 and 108000 is presented. Line, soft-SAFT predictions; symbols, experimental data taken from the literature. ${ }^{36}$

of the molecules, since the binary parameter is a correction to this energy of interaction. Results obtained with PC-SAFT also with a binary parameter present a similar behavior, although the predictions deteriorate near the LCST, which are slightly overestimated compared to soft-SAFT calculations. It is remarkable the description provided with both equations with just one binary parameter and very close to unity $\left(\xi_{i j}\right.$ soft-SAFT $=0.9675$; $\xi_{i j}$ PC-SAFT $\left.=\left(1-k_{i j}\right)=0.9730\right)$.

3.2.4. Polyethylene/1-Pentanol. The use of associating solvents adds extra complexity to the system. To check the accuracy of the equation, we have investigated the description of a mixture of polyethylene $\left(M_{\mathrm{n}}=20000 \mathrm{~g} / \mathrm{mol}\right)$ with pentanol ${ }^{38}$ using the soft-SAFT EoS. The results obtained with the parameters provided in Tables 1 and 2 are shown in Figure 8. A binary interaction parameter with a value of 0.9873 was needed, as otherwise the phase coexistence would be underestimated. The data available are rather scarce, making difficult to assess the accuracy of the soft-SAFT EoS.

3.2.5. Polyethylene/Ethylene. The description of the phase equilibria of the polymer with its monomer is of the utmost importance, as they coexist throughout the whole manufacturing process. It starts in the reaction itself, but the most important 


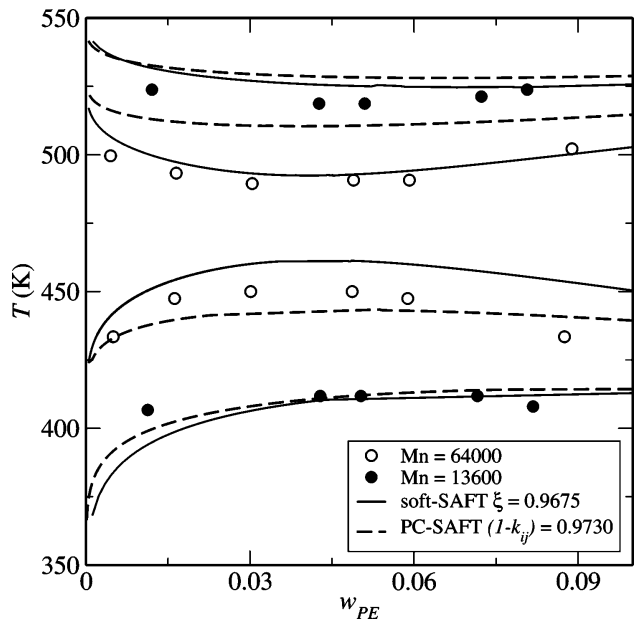

Figure 7. Liquid-liquid phase equilibria modeled with the soft-SAFT EoS (full lines) and the PC-SAFT EoS (dashed lines) of a mixture of polyethylene and butyl acetate at a constant pressure of $0.1 \mathrm{MPa}$, with a fit binary parameter to literature data (symbols). ${ }^{37}$

Table 1. Molecular Parameters of the SAFT EoSs for the Polyethylene Polymers

\begin{tabular}{rcccll}
\hline $\begin{array}{c}\boldsymbol{M}_{\mathrm{n}}(\mathrm{g} / \\
\text { mol })\end{array}$ & model & $m$ & $\sigma(\AA)$ & \multicolumn{1}{c}{$\begin{array}{c}\epsilon / k_{\mathrm{B}} \\
(\mathrm{K})\end{array}$} & \multicolumn{1}{c}{ reference } \\
\hline 16000 & soft-SAFT & 408.6 & 4.077 & 294.7 & eq 2 \\
16000 & soft-SAFT & 408.6 & 4.077 & 309.4 & eqs 2a, 2b, and 4 \\
16000 & PC-SAFT & 411.93 & 4.064 & 272.45 & eq 3 \\
16000 & PC-SAFT & 420.8 & 4.0217 & 249.5 & 20 \\
76000 & soft-SAFT & 1938.6 & 4.078 & 294.8 & eqs 2a, 2b, and 4 \\
108000 & soft-SAFT & 2754.6 & 4.078 & 294.9 & eqs 2a, 2b, and 4 \\
15000 & soft-SAFT & 383.1 & 4.078 & 294.6 & eqs 2a, 2b, and 4 \\
64000 & soft-SAFT & 1632.6 & 4.078 & 294.8 & eqs 2a, 2b, and 4 \\
13600 & soft-SAFT & 347.4 & 4.077 & 294.6 & eqs 2a, 2b, and 4 \\
20000 & soft-SAFT & 510.6 & 4.078 & 294.7 & eqs 2a, 2b, and 4 \\
31700 & soft-SAFT & 809.0 & 4.078 & 294.8 & eqs 2a, 2b, and 4
\end{tabular}

Table 2. Molecular Parameters of the Soft-SAFT EoS for the Solvents

\begin{tabular}{lcccccl}
\hline & $m$ & $\sigma(\AA)$ & $\begin{array}{c}\epsilon / k_{\mathrm{B}} \\
(\mathrm{K})\end{array}$ & $\begin{array}{c}k_{\mathrm{HB}} \\
\left(\AA^{3}\right)\end{array}$ & $\begin{array}{c}\epsilon_{\mathrm{HB}} / k_{\mathrm{B}} \\
(\mathrm{K})\end{array}$ & \multicolumn{1}{c}{ ref } \\
\hline$n$-pentane & 2.497 & 3.901 & 246.6 & & & 29 \\
$n$-hexane & 2.832 & 3.929 & 254.4 & & & 29 \\
butyl acetate & 3.728 & 3.634 & 262.7 & & & this work \\
1-pentanol & 2.420 & 4.051 & 283.7 & 2250 & 3450 & 29 \\
ethylene & 1.431 & 3.599 & 187.8 & & & this work
\end{tabular}

step is the separation of unreacted monomer from the polymer. This is usually done in flash vessels specifically made for this purpose. To predict the flash operation, accurate phase equilibria data are needed. We have also described the gas-polymer phase equilibria with the soft-SAFT EoS. The results are depicted in Figure 9 , where the solubility of ethylene in polyethylene $\left(M_{\mathrm{n}}\right.$ $=31700)^{39}$ is represented. It is observed that pure component parameters clearly underestimate the pressures, while a binary interaction parameter $(0.900)$ provides good estimations of the vapor pressure of the mixtures. Even with the use of this binary parameter the mixture behavior is not exactly described by the soft-SAFT EoS; the equation is not able to capture the shape of the solubility curves. An explanation is the fact that the softSAFT EoS does not consider the possible degree of crystallization present in the polymer. This is particularly important in gas-polymer equilibria as the polymer is not being dissolved, but instead, it is the gas that is dissolving into the polymer. In pure polyethylene, the fraction of polymer that is in crystal form can reach $100 \%$ if the polymer is linear. At the modeled conditions, that is, high concentration of polymer, it is possible that some of the polymer exists in the crystal form. This is not

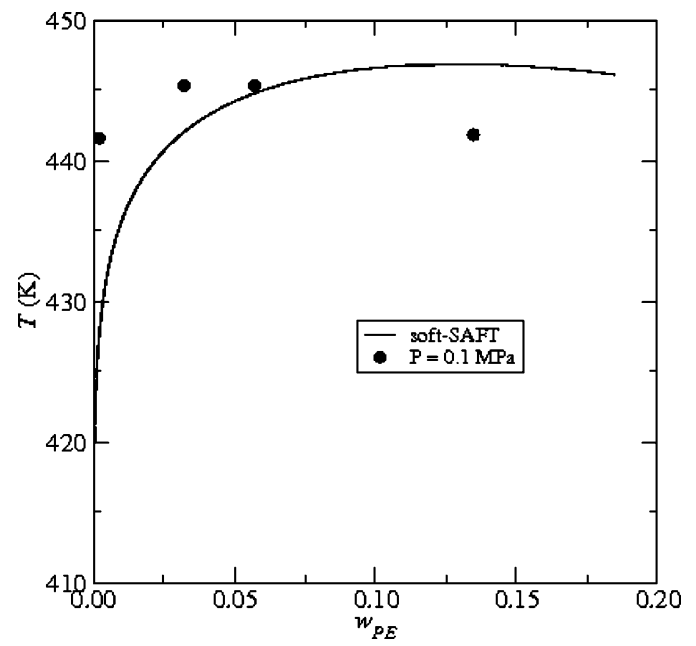

Figure 8. Phase behavior description of the soft-SAFT EoS of a polyethylene with a number molecular weight of 20000 mixed with 1-pentanol. A binary interaction parameter was fit to experimental data reported in the literature. ${ }^{38}$ Lines, soft-SAFT EoS; symbols, experimental data.

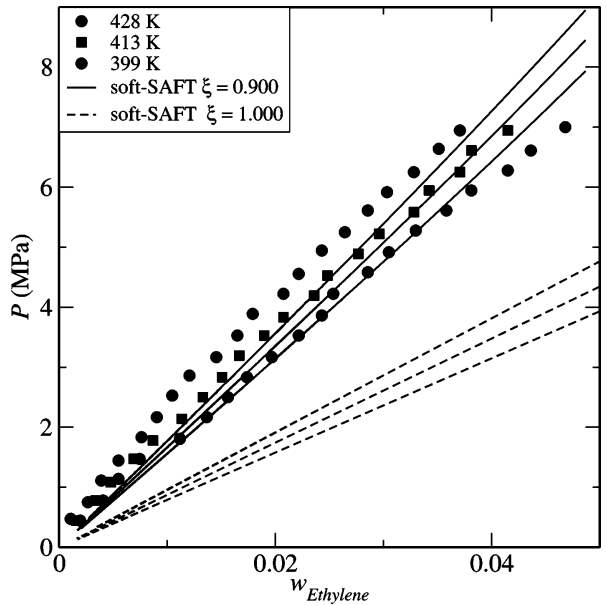

Figure 9. Gas solubility of ethylene in polyethylene $\left(M_{\mathrm{n}}=31700\right)$. Full lines represent the soft-SAFT model with an adjusted binary interaction parameter, and dotted lines are the calculations of the mentioned model without binary interaction parameters. The experimental data were extracted from the literature. ${ }^{39}$

taken into account explicitly in the soft-SAFT (and PC-SAFT) EoS.

\section{Conclusions}

We have shown here the accuracy of the soft-SAFT EoS to model the phase equilibria of polyethylene with several solvents, including pentane, hexane, butyl acetate, pentanol, and ethylene. Results obtained with soft-SAFT were compared with experimental data and with the performance of the PC-SAFT EoS for the same systems.

We have circumvented one of the main drawbacks of using EoSs to model polymer systems, namely the difficulty to obtain the pure polymer parameters. This has been done with two alternative ways: (1) by using the molecular parameters obtained for the $n$-alkane series to model polyethylene and (2) by proposing a new correlation of the energy parameter with the molecular weight, while keeping the size and chain length parameters from the correlation of the $n$-alkane series. The new correlation is able to accurately describe the behavior of polymer mixtures with various solvents, in better agreement with experimental data than the original $n$-alkanes correlation. 
The accuracy of the soft-SAFT EoS for these polymeric systems has been proved in several ways: the equation was able to capture the vapor-liquid dependence with temperature, the simultaneous existence of upper critical solution temperature and lower critical solution temperature for the mixture with butyl acetate, and the gas solubility in polyethylene. The polymer/nalkane systems were predicted from pure component parameters, while one energy binary parameter, close to unity, was needed in order to describe the polymer with butyl acetate, 1-pentanol, and ethylene binary mixtures. The comparison between results obtained with soft-SAFT and PC-SAFT shows that both equations provide similar results in most of the cases, being soft-SAFT slightly more accurate for some particular mixtures.

This work is a step forward on systematically developing sound molecular models for the description of polymer systems. The availability of transferable parameters will empower then with predictive capabilities for other polymer/solvent mixtures, including multicomponent solutions. Along the line of this work, a good method to obtain transferable parameters would be to use the properties (and parameters) of the monomer forming the chain and extend them to the polymer molecule. This would be particularly useful for polymers made of large monomers such as polystyrene or poly(methyl methacrylate) as their single properties would be more similar to the properties of the polymer segments considered by a molecular model such as then one used by SAFT-based equations of state.

Acknowledgment. N. Pedrosa acknowledges the "Fundação para a Ciência e Tecnologia" PhD scholarship (SFRH/BD/ 11041/2002). Financial support for this work as been provided by the Portuguese government through project POCTI/43356/ 2001, by the Spanish government under project CTQ200500296/PPQ, and by the Generalitat de Catalunya (SGR200500288).

\section{References and Notes}

(1) Scott, R. L.; van Konynenburg, P. H. Discuss. Faraday Soc. 1970, 49, 87-97.

(2) Huggins, M. L. J. Chem. Phys. 1942, 10, 51-61.

(3) Sanchez, I. C.; Lacombe, R. H. J. Phys. Chem. 1976, 80, 2352-2362.

(4) Sanchez, I. C.; Lacombe, R. H. Macromolecules 1978, 11, 11451156.

(5) Nagya, I.; Loos, T. D.; Krenz, R.; Heidemann, R. J. Supercrit. Fluids 2006, 37, 115-124.

(6) Challa, V. V.; Visco, D. P., Jr. J. Cell. Plast. 2005, 41, 563-588.
(7) Wertheim, M. S. J. Stat. Phys. 1984, 35, 19-34.

(8) Wertheim, M. S. J. Stat. Phys. 1984, 35, 35-47.

(9) Wertheim, M. S. J. Stat. Phys. 1986, 42, 459-476.

(10) Wertheim, M. S. J. Stat. Phys. 1986, 42, 477-492.

(11) Chapman, W. G.; Gubbins, K. E.; Jackson, G.; Radosz, M. Fluid Phase Equilib. 1989, 52, 31-38.

(12) Huang, S. H.; Radosz, M. Ind. Eng. Chem. Res. 1990, 29, 22842294.

(13) Villegas, A. G.; Galindo, A.; Whitehead, P. J.; Mills, S. J.; Jackson, G.; Burgess, A. N. J. Chem. Phys. 1997, 106, 4168-4186.

(14) Blas, F. J.; Vega, L. F. Mol. Phys. 1997, 92, 135-150.

(15) Gross, J.; Sadowski, G. Ind. Eng. Chem. Res. 2001, 40, 1244-1260.

(16) Chen, S. J.; Economou, I. G.; Radosz, M. Macromolecules 1992, 25 , 4987-4995.

(17) Wu, C.-S.; Chen, Y.-P. Fluid Phase Equilib. 1994, 100, 103-119.

(18) Ghonasgi, D.; Chapman, W. G. AIChE J. 1994, 40, 878-887.

(19) Koak, N.; Heidemann, R. A. Ind. Eng. Chem. Res. 1996, 35, 43014309.

(20) Gross, J.; Sadowski, G. Ind. Eng. Chem. Res. 2002, 41, 1084-1093.

(21) Sadowski, G. Macromol. Symp. 2004, 206, 333-346.

(22) Solms, N. v.; Michelsen, M. L.; Kontogeorgis, G. M. Ind. Eng. Chem. Res. 2003, 42, 1098-1105.

(23) Kouskoumvekaki, I. A.; Solms, N. v.; Michelsen, M. L.; Kontogeorgis, G. M. Fluid Phase Equilib. 2004, 215, 71-78.

(24) Kouskoumvekaki, I. A.; Solms, N. v.; Lindvig, T.; Michelsen, M. L.; Kontogeorgis, G. M. Ind. Eng. Chem. Res. 2004, 43, 2830-2838.

(25) Solms, N. v.; Kouskoumvekaki, I. A.; Lindvig, T.; Michelsen, M. L.; Kontogeorgis, G. M. Fluid Phase Equilib. 2004, 222, 87-93.

(26) Solms, N. v.; Michelsen, M. L.; Kontogeorgis, G. M. Ind. Eng. Chem. Res. 2005, 44, 3330-3335.

(27) Pàmies, J. C.; Vega, L. F. Mol. Phys. 2002, 100, 2519-2529.

(28) Florusse, L. J.; Pàmies, J. C.; Vega, L. F.; Peters, C. J.; Meijer, H. AIChE J. 2003, 49, 3260-3269.

(29) Pàmies, J. C.; Vega, L. F. Ind. Eng. Chem. Res. 2001, 40, 25322543.

(30) Müller, E. A.; Gubbins, K. E. Ind. Eng. Chem. Res. 2001, 40, 21932211.

(31) Economou, I. G. Ind. Eng. Chem. Res. 2002, 41, 953-962.

(32) Ghosh, A.; Ting, P. D.; Chapman, W. G. Ind. Eng. Chem. Res. 2005, 43, 6222-6230.

(33) Danner, R. P.; High, M. S. Handbook of Polymer Solution Thermodynamics; DIPPR, AIChE Press: New York, 1993.

(34) Kiran, E.; Zhuang, W. Polymer 1992, 33, 5259-5263.

(35) Surana, R.; Danner, R.; De Haan, A.; Beckers, N. Fluid Phase Equilib. 1997, 139, 361-370.

(36) Chen, X.; Yasuda, K.; Sato, Y.; Takishima, S.; Masuoka, H. Fluid Phase Equilib. 2004, 215, 105-115.

(37) Kuwahara, N.; Saeki, S.; Chiba, T.; Kaneko, M. Polymer 1974, 15 $777-781$

(38) Nakajima, A.; Fujiwara, H.; Hamada, F. J. Polym. Sci., Part A2 1966, 4, 507.

(39) Hao, W.; Elbro, H. S.; Alessi, P. Polymer Solution Data Collection: Chemistry Data Series; DECHEMA: Frankfurt, 1992; Vol. XIV, Part 1.

MA060584A 